\title{
Genomic dissection of the most prevalent Listeria monocytogenes clone, sequence type ST87, in China
}

\author{
Yan Wang ${ }^{1}$, Lijuan Luo ${ }^{2}$, Qun $\mathrm{Li}^{3}$, Hong Wang ${ }^{3}$, Yiqian Wang ${ }^{1}$, Hui Sun ${ }^{1}$, Jianguo Xu' ${ }^{1}$, Ruiting Lan ${ }^{2^{*}}$ and \\ Changyun $Y e^{1 *}$
}

\begin{abstract}
Background: Listeria monocytogenes consists of four lineages that occupy a wide variety of ecological niches. Sequence type (ST) 87 (serotype 1/2b), belonging to lineage I, is one of the most common STs isolated from food products, food associated environments and sporadic listeriosis in China. Here, we performed a comparative genomic analysis of the L. monocytogenes ST87 clone by sequencing 71 strains representing a diverse range of sources, different geographical locations and isolation years.

Results: The core genome and pan genome of ST87 contained 2667 genes and 3687 genes respectively. Phylogenetic analysis based on core genome SNPs divided the 71 strains into 10 clades. The clinical strains were distributed among multiple clades. Four clades contained strains from multiple geographic regions and showed high genetic diversity. The major gene content variation of ST87 genomes was due to putative prophages, with eleven hotspots of the genome that harbor prophages. All strains carry an intact CRISRP/Cas system. Two major CRISPR spacer profiles were found which were not clustered phylogenetically. A large plasmid of about $90 \mathrm{~Kb}$, which carried heavy metal resistance genes, was found in 32.4\% (23/71) of the strains. All ST87 strains harbored the Listeria pathogenicity island (LIPI)-4 and a unique 10-open read frame (ORF) genomic island containing a novel restriction-modification system.

Conclusion: Whole genome sequence analysis of L. monocytogenes ST87 enabled a clearer understanding of the population structure and the evolutionary history of ST87 L. monocytogenes in China. The novel genetic elements identified may contribute to its virulence and adaptation to different environmental niches. Our findings will be useful for the development of effective strategies for the prevention and treatment of listeriosis caused by this prevalent clone.
\end{abstract}

Keywords: Listeria monocytogenes, Whole genome sequencing, Genomic comparative analysis, ST87

\section{Background}

Listeria monocytogenes is an important foodborne bacterial pathogen, which is able to switch from a saprotrophic lifestyle to an intracellular pathogenic lifestyle [1]. The bacterium has been isolated from many different natural environments (such as soil, water and plant materials), various food products and food processing

\footnotetext{
*Correspondence: r.lan@unsw.edu.au; yechangyun@icdc.cn

${ }^{2}$ School of Biotechnology and Biomolecular Sciences, University of New South Wales, Sydney, NSW 2052, Australia

'State Key Laboratory of Infectious Disease Prevention and Control, National Institute for Communicable Disease Control and Prevention, Collaborative Innovation Center for Diagnosis and Treatment of Infectious Diseases, Chinese Center for Disease Control and Prevention, Beijing 102206, China Full list of author information is available at the end of the article
}

environments [2]. L. monocytogenes causes listeriosis, which is a severe invasive infection manifesting with sepsis, meningoencephalitis or pregnancy loss, and can be fatal in immunocompromised individuals. Sporadic listeriosis and outbreaks have been reported that were linked to various food products, such as ready-to-eat (RTE) food, raw meat, pasteurized milk, ice cream, fruit, and vegetable [3-5].

L. monocytogenes consists of at least four lineages, of which lineages I and II encompass the majority of isolates [6]. Within these two common lineages, the populations have been further divided into sequence types (STs) or clonal complexes (CCs) by multi-locus sequence typing (MLST). Previous studies have found that

(c) The Author(s). 2019 Open Access This article is distributed under the terms of the Creative Commons Attribution 4.0 International License (http://creativecommons.org/licenses/by/4.0/), which permits unrestricted use, distribution, and 
some clones (STs or CCs) such as CC1, CC2, CC4 and CC6 were strongly associated with sporadic or outbreak listeriosis, whereas some other clones CC9 and CC121 were strongly associated with food product contamination but less with human infections [7]. The difference in prevalence of causing human infections may be associated with difference in virulence. Some clones are known to carry genes conferring higher virulence such as Listeria pathogenicity island (LIPI)-3 and LIPI-4 [7, 8], whereas other clones (e.g. ST121) may carry genes better for environmental survival, for example, the stress survival islet (SSI)-1 and SSI-2 $[9,10]$.

In China, L. monocytogenes contamination of food products has been found to be quite extensive [11-13]. However, the prevalence of this pathogen in clinical cases has been seldom reported due to the lack of a good listeriosis surveillance system in China [14]. Our previous studies found that L. monocytogenes ST87 was the most common ST in human infections in China and was also commonly found in food products and food associated environments [13-16]. Other studies confirmed our findings $[17,18]$. However, ST87 has been rarely reported in other countries with the exception of Spain, where two epidemiological unrelated outbreaks caused by ST87 occurred in 2013 and 2014 [19].

Whole-genome sequencing (WGS) can provide comprehensive knowledge on genetic determinants of the reponse to stress conditions and virulence factors [7, 20]. WGS can elucidate the population structure within a single sequence type or clonal complex more clearly $[21,22]$. In this study, we performed WGS analysis on 71 ST87 L. monocytogenes strains, which spanned a period of 15 years (from 2001 to 2015), and represented 10 isolation regions (Beijing and Shanghai city, Anhui, Fujian, Guangdong, Henan, Hubei, Jiangsu, Sichuan and Zhejiang province), and seven types of sources (aquatic food, cooked food, poultry, raw meat, vegetables, environment and patients) (Additional file 5: Table S1). We aimed to obtain a clearer understanding of the microevolution of the ST87 clone and putative genetic elements responsible for the adaptation and persistence in enrivonmental niches, and factors facilitating human infections to enable more effective strategies of prevention and control of this foodborne disease in China.

\section{Results}

The general genomic features of $L$. monocytogenes ST87 To investigate the genomic diversity of $L$. monocytogenes ST87 in China, we sequenced one ST87 strain fully and obtained draft genomes for 70 strains. ST87 strain ICDC-LM188 obtained from a patient was sequenced using Illumina Hiseq 2000 and Applied Biosystems 3730 DNA Analyzer to obtain its complete genome and was used as reference genome in this study. ICDC-LM188 has a 2,982,685 bp single chromosome with $\mathrm{G}+\mathrm{C}$ content of 37.97\% (Fig. 1). The genome contained 2919 genes including 2882 coding sequences (CDS), 67 tRNA genes, six $16 \mathrm{~S}-5 \mathrm{~S}-23 \mathrm{~S}$ operons and 11 pseudogenes. For the 70 draft genomes, they were sequenced to the depth of 100X coverage on average. The scaffolds of draft genome assemblies varied from $2.8 \mathrm{Mb}$ to $3.1 \mathrm{Mb}$. The number of predicted genes ranged from 2854 to 3187 (Additional file 6: Table S2). The ST87 pan and core genome contained 3687 genes and 2677 genes respectively. The ST87 core genome is $13.7 \%$ larger than the $L$. monocytogenes core genome (2354 genes), while the ST87 pan genome is $15.9 \%$ smaller than the species pan genome (4383 genes) [23]. The three most abundant functional categories of the core genes were carbohydrate transport and metabolism (G), transcription (K) and amino acid transport and metabolism (E). While the top three functional categories of the accessory genes were replication, recombination and repair $(\mathrm{L})$, mobilome $(\mathrm{X})$ and transcription (K) (Additional file 1: Figure S1). A large fraction of the gene content (84.0-93.7\%) in each strain were core genes.

\section{Single nucleotide polymorphism (SNP) analysis and phylogenetic analysis}

A comparison across all ST87 strains sequenced in this study, using strain ICDC-LM188 as the reference, revealed a total of 5415 SNP positions, of which 1410 (26.0\%) were nonsynonymous, 3775 (69.7\%) synonymous and $230(4.3 \%)$ in intergenic regions. Of all the SNPs, 644 were located in the core genes $(n=478,17.9 \%)$. Interestingly, almost two thirds of the SNPs $(n=437)$ located in the core genes were nonsynonymous, which was very different from the SNPs located on the whole genome. The core genes that harbored SNPs were classified according to the COG database. Based on the predictive function, the largest ratio of nonsynonymous to synonymous mutation occurred in the genes involved in general function prediction only (R), followed by lipid transport and metabolism (I) and inorganic ion transport and metabolism (P) (Table 1).

SNPs present in the core genome were used to infer the phylogenetic relationship of the 71 sequenced ST87 strains using the maximum likelihood method (Fig. 2). Ten clades can be discerned from the SNP-based genome tree with strong bootstrap support. Six of the 10 clades contained strains from the same geographic source. Clade 1, 5 and 9 consisted of only two strains with identical geographic source, but from different years or sources. Clade 8 consisted of five clinical strains in one maternalfetal case and its closely related strain from a food source. Clade 10 contained 10 strains isolated from a single market in Sichuan in 2015. Four clades contained strains from more than one geographic source and showed higher 


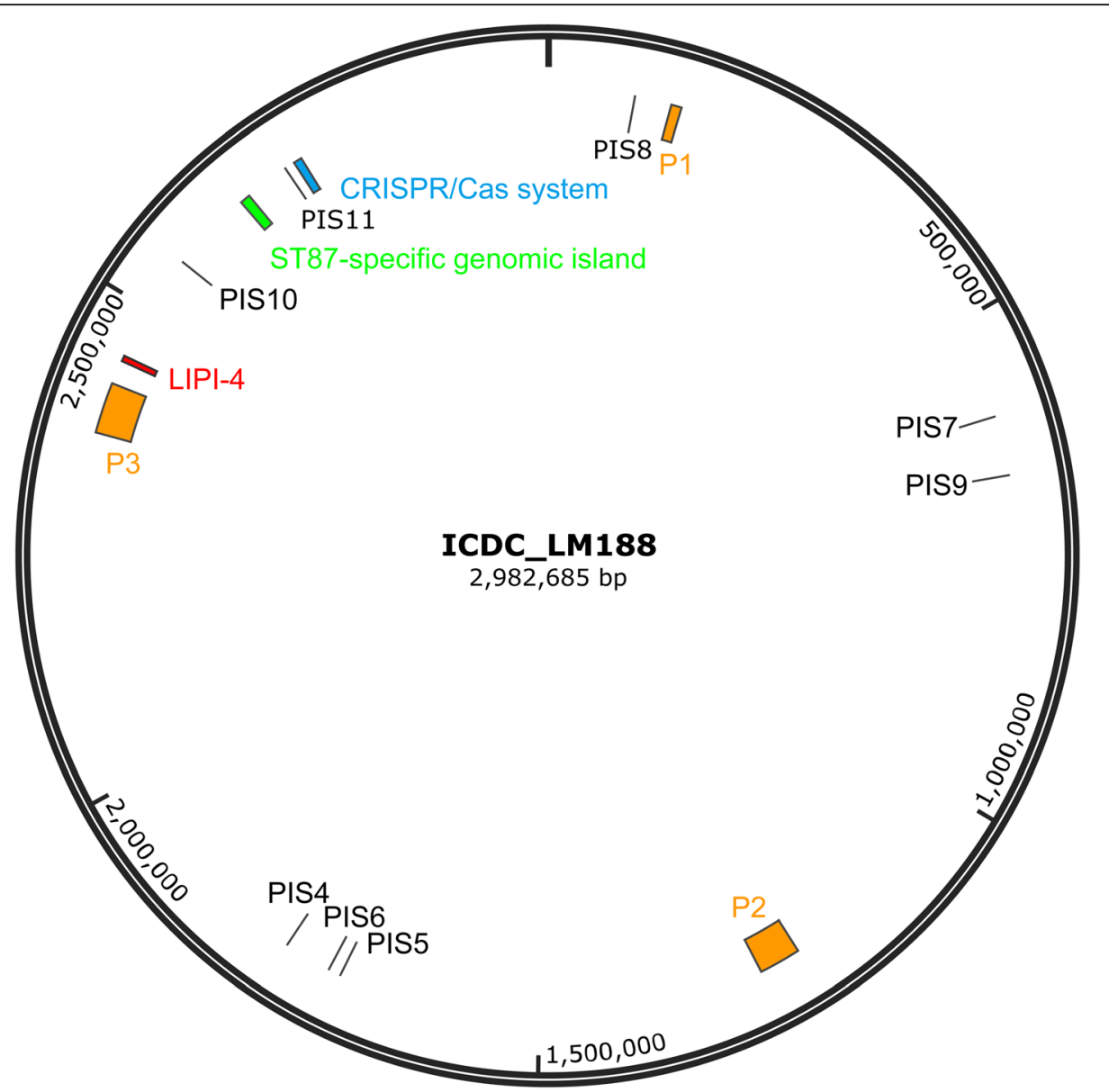

Fig. 1 A circular representation of the strain ICDC-LM188 chromosome and the key genetic features of the genome. Putative prophage regions (P1-P3) were marked as orange blocks; LIPI-4, the ST87-specific genomic island and the CRISPR/Cas system were marked as red, green and blue blocks, respectively. The integrated sites of putative prophages (P4 to P11) in other ST87 isolates but not in the strain ICDC-LM188 are labeled as PIS4 to PIS11

genetic diversity. However, many branches showed low bootstrap support with a high degree of uncertainty. BEAST analysis indicated that ST87 arose approximately 200 years ago and expanded quickly with a star phylogeny at the base of the tree, which was also reflected on the low bootstrap values of many nodes of the genome tree. To investigate the relationships between the Chinese and nonChinese ST87 strains, seven ST87 L. monocytogene genomes, of which six were from the USA and one from Austria, were also added to the phylogenetic analysis. The non-Chinese strains were distributed among the Chinese strains with three strains from the USA grouped together as a separate clade (Additional file 2: Figure S2).

\section{Prophages in ST87 L. monocytogenes strains}

Using PHAge Search Tool Enhanced Release (PHASTER) online software, three putative prophages have been identified from the complete genome of ICDCLM188 (Fig. 1). Putative prophage 1 (P1) was $10.7 \mathrm{~Kb}$ in size inserted upstream of the $5^{\prime}$-nucleotidase gene (ICDCLM188 locus tag: A6K41_00700 [lmo0130 in EGD-e]). P1 has been found as an incomplete prophage previously and was named monocin, which harbored a lma operon (lmaDCBA) and conserved across Lineage I and II strains [21, 22]. Putative prophage 2 (P2) was $43.1 \mathrm{~Kb}$ in size with 58 predicted genes, and was inserted downstream of tRNAArg-TCT. P2 shared 29 genes with Listeria phage LP-101 [24]. Putative prophage 3 (P3) was $52.5 \mathrm{~Kb}$ in size with 57 genes, and was inserted into comK. P3 shared 34 genes with Listeria phage A006. P1 was shared by all sequenced ST87 strains, while P2 and P3 were present in 44 and 62 of the 71 ST87 strains respectively (Fig. 2). It is noteworthy that the genetic contents of P2 and P3 exhibited high diversity among the ST87 L. monocytogenes strains.

In addition, another eight putative prophage regions (P4 - P11) were identified in a small proportion of ST87 strains but absent in strain ICDC-LM188. The prophage integration sites of P4 - P11 in the ICDC-LM188 
Table 1 Nonsynonymous- and synonymous-SNPs identified within core genes by COG category

\begin{tabular}{|c|c|c|c|}
\hline COG category & Nonsynonymous & Synonymous & Ratio \\
\hline $\begin{array}{l}\text { Energy production and } \\
\text { conversion }(C)\end{array}$ & 14 & 9 & 1.56 \\
\hline $\begin{array}{l}\text { Cell cycle control, cell division, } \\
\text { chromosome partitioning (D) }\end{array}$ & 5 & 3 & 1.67 \\
\hline $\begin{array}{l}\text { Amino acid transport and } \\
\text { metabolism (E) }\end{array}$ & 35 & 14 & 2.50 \\
\hline $\begin{array}{l}\text { Nucleotide transport and } \\
\text { metabolism (F) }\end{array}$ & 10 & 3 & 3.33 \\
\hline $\begin{array}{l}\text { Carbohydrate transport } \\
\text { and metabolism (G) }\end{array}$ & 52 & 24 & 2.17 \\
\hline $\begin{array}{l}\text { Coenzyme transport and } \\
\text { metabolism }(\mathrm{H})\end{array}$ & 14 & 10 & 1.40 \\
\hline $\begin{array}{l}\text { Lipid transport and } \\
\text { metabolism (I) }\end{array}$ & 7 & 1 & 7.00 \\
\hline $\begin{array}{l}\text { Translation, ribosomal } \\
\text { structure and biogenesis }(J)\end{array}$ & 29 & 9 & 3.22 \\
\hline Transcription (K) & 37 & 15 & 2.47 \\
\hline $\begin{array}{l}\text { Replication, recombination } \\
\text { and repair }(\mathrm{L})\end{array}$ & 46 & 61 & 0.75 \\
\hline $\begin{array}{l}\text { Cell wall/membrane/envelope } \\
\text { biogenesis }(\mathrm{M})\end{array}$ & 20 & 5 & 4.00 \\
\hline Cell motility (N) & 6 & 6 & 1.00 \\
\hline $\begin{array}{l}\text { Posttranslational modification, } \\
\text { protein turnover, chaperones }(\mathrm{O})\end{array}$ & 16 & 3 & 5.33 \\
\hline $\begin{array}{l}\text { Inorganic ion transport and } \\
\text { metabolism }(P)\end{array}$ & 19 & 3 & 6.33 \\
\hline $\begin{array}{l}\text { Secondary metabolites } \\
\text { biosynthesis, transport } \\
\text { and catabolism (Q) }\end{array}$ & 4 & 2 & 2.00 \\
\hline $\begin{array}{l}\text { General function prediction } \\
\text { only }(R)\end{array}$ & 29 & 3 & 9.67 \\
\hline Function unknown (S) & 30 & 15 & 2.00 \\
\hline $\begin{array}{l}\text { Signal transduction } \\
\text { mechanisms (T) }\end{array}$ & 10 & 3 & 3.33 \\
\hline $\begin{array}{l}\text { Intracellular trafficking, secretion, } \\
\text { and vesicular transport }(U)\end{array}$ & 1 & 0 & \\
\hline Defense mechanisms ( $V$ ) & 16 & 3 & 5.33 \\
\hline Extracellular structures (W) & 0 & 0 & \\
\hline $\begin{array}{l}\text { Mobilome: prophages, } \\
\text { transposons }(X)\end{array}$ & 1 & 1 & 1.00 \\
\hline
\end{tabular}

The top three ratios of nonsynonymous- to synonymous- SNPs harbored core genes were marked as red

genome were shown in Fig. 1. Prophage P4 was located between the gene encoding FosX/FosE/FosI family fosfomycin resistance thiol transferase (ICDC-LM188 locus tag: A6K41_08870 [lmo1702 in EGD-e]) and the gene encoding 23S rRNA (uracil-5-)-methyltransferase (RumA, ICDC-LM188 locus tag: A6K41_08875 [lmo1703 in EGDe]). Six ST87 strains contained this prophage which was represented by two variants, $\phi$ LMST87_001 (37.7 Kb) and фLMST87_002 (40.6 Kb). Sequence coverage was 92\% with $96 \%$ nucleotide identity when the two variants were

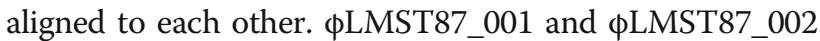
showed high similarity to the prophage present in Erysipelothrix sp. strain LV19, with 95\% and 96\% nucleotide identity, and 99\% and 96\% coverage respectively. Both prophages encode an $\mathrm{HNH}$ family endonuclease gene and two adjoining DNA methyltransferases genes (adenineand cytosine-specific, respectively). The R-M system might play a role of excluding other phages when the host encountered exogenous phage infections [25]. They also contained two virulence related proteins. This phage integration site has been reported in a subset of ST204 $L$. monocytogenes strains, which harbored a $56.9 \mathrm{~Kb} \phi \mathrm{RNA}-$ MT [21]. However, the gene contents of prophages in ST87 (фLMST87_001 and фLMST87_002) and in ST204 ( $\phi$ RNA-MT) were totally different as the alignment between them showed only $34 \%$ and $36 \%$ query coverage, respectively. P5, only found in strain LM0146, was integrated into the 3 ' end of tsf which encodes the elongation factor EF-Ts (ICDC-LM188 locus tag: A6K41_08510 [lmo1657 in EGD-e]). It showed 96\% nucleotide identity with $85 \%$ coverage towards the Listeria phage B054, and was inserted at the same phage integration site as phage B054. P6, also named as QLMST87_003, is located downstream of the gene encoding methionine adenosyltransferase (MAT, ICDC-LM188 locus tag: A6K41_08565 [lmo1664 in EGD-e]) and is $43.1 \mathrm{~Kb}$ in size. It contained 74 genes. Only two strains, LM0441 and LM0449, harbored LMST87_003 which showed no similarity with any reported Listeria phage. P7 is a putative prophage remnant and was inserted into the upstream of 50S rRNA methyltransferase (ICDCLM188 locus tag: A6K41_02920 [lmo0581 in EGD-e]). It was found in four closely related isolates (LM1681, LM1682, LM1685 and LM1689).

We also found four putative prophage regions (P8 P11) which were typically inserted into chromosomal loci adjacent to tRNA genes, including tRNA-CTT-Lys (ICDC-LM188 locus tag: A6K41_00440, the tRNA-Lys after lmo0078), tRNA-CGA-Ser (ICDC-LM188 locus tag: A6K41_03215, the tRNA-Ser after lmo0670), tRNACCG-Arg (ICDC-LM188 locus tag: A6K41_12845, the tRNA-Arg after lmo2466) and tRNA-GGT-Thr (ICDCLM188 locus tag: A6K41_13515, the tRNA-Thr after lmo2589). P10, named фLMST87_004, was assembled into a complete prophage sequence in strain LM1296. It was $43.6 \mathrm{~kb}$ in size and has 74 predicted genes. фLMST87_004 showed 90\% nucleotide identity with Listeria phage A118 with $65 \%$ coverage.

\section{Plasmids in ST87 L. monocytogenes strains}

We previously identified a plasmid (pLM1686) in strain LM1686 which is homologous to the plasmid from $L$. monocytogenes strain 08-5578 (serotype 1/2a, ST8), with 
(See figure on previous page.)

Fig. 2 Phylogenetic tree of the 71 ST87 strains. The tree was constructed using the maximum likelihood method based on core genome SNPS. The strains isolated from different regions were marked with different colors (red for Sichuan, blue for Beijing, green for Zhejiang, brown for Shanghai and blank for Fujian, Henan, Hubei and Jiangsu). The strains isolated from clinical cases were highlighted. The strains harbored the plasmid were labeled with a red solid dot. The corresponding putative prophage and the profile of spacers were shown alongside the dendrogram on the right

99\% identity in alignments covering 73\% of pLM5578. Detailed analysis of pLM1686 has been presented elsewhere [16]. We further determined to which the phylogenetic group pLM1686 from ST87 belonged to among the plasmids in L. monocytogenes. We used RepA amino acid sequences from all 22 known plasmids to construct the phylogenetic tree, using the RepA of pOX2 from Bacillus anthracis as an outgroup (Fig. 3b). The plasmids clustered into four groups, and pLM1686 belonged to group 2. The plasmid genome contained cadmium resistance cadAC genes and also a putative multicopper oxidase gene and copper transporter gene $\operatorname{cop} B$, but no genes associated with virulence or antimicrobial resistance [16].
pLM1686 is absent in ICDC-LM188. However, plasmids very similar to pLM1686 were found in 22 other ST87 strains sequenced in this study. All plasmids had a similar size of approximately $91 \mathrm{~kb}$. Comparison of the common regions among the 23 plasmid sequences identified only eight SNPs suggesting that the plasmids were very closely related. A phylogenetic tree of the plasmids showed five plasmid types with the majority (18 out of 23 ) belonging to one type with identical or near identical sequences (Fig. 3a). All plasmid-positive strains except four were clustered together on the core genome tree. Six plasmid positive strains, LM0429, LM0422, LM0476, LM0146, LM0099 and LM0208 were located on different branches of the tree, suggesting independent acquisitions of the plasmid.

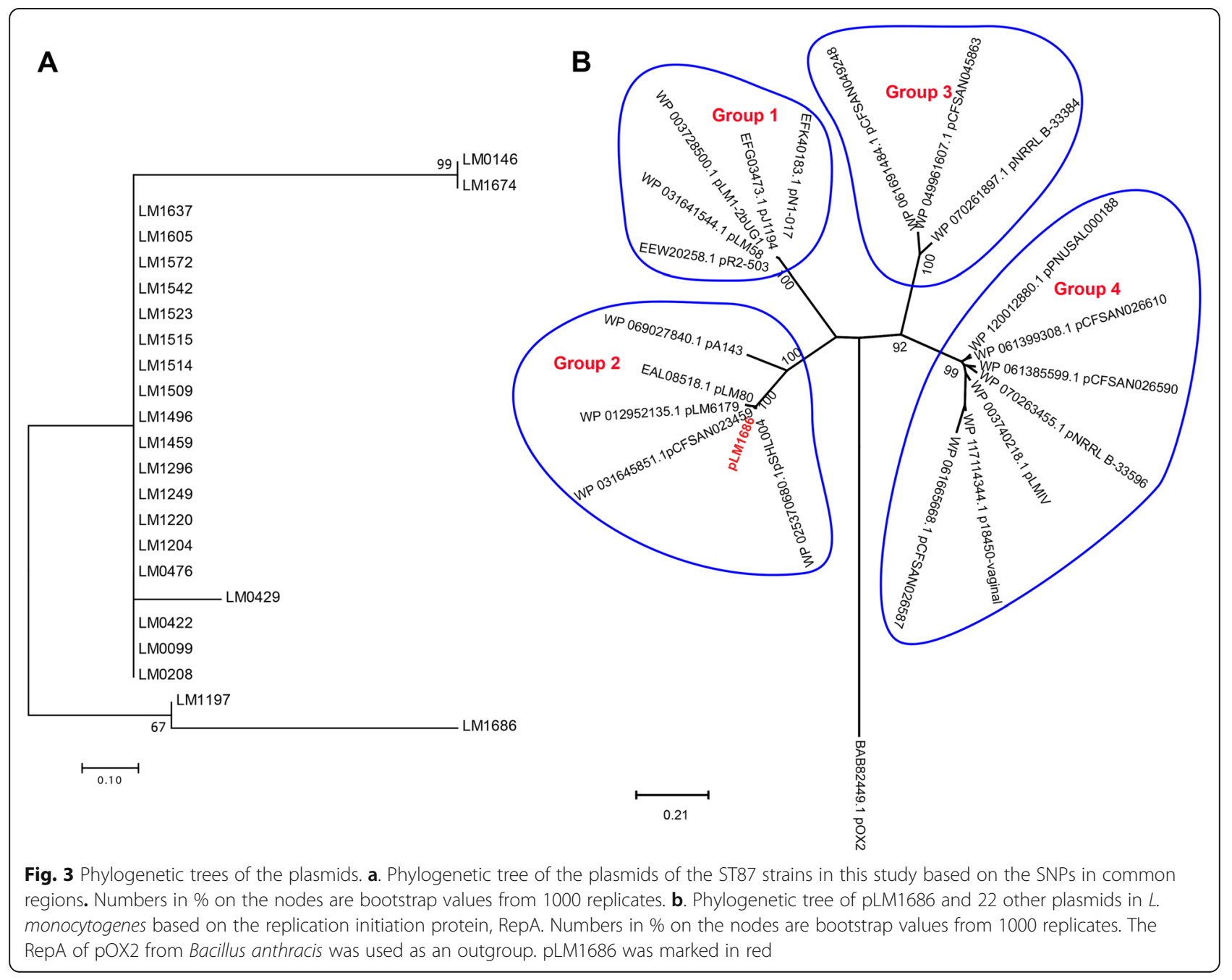




\section{CRISPR systems in ST87 strains}

The chromosome of strain ICDC-LM188 contains two CRISPR systems. Locus 1 is a remnant of a CRISPR system without any cas genes. The content of locus 1 showed $100 \%$ nucleotide identity with that of strain $1 / 2 \mathrm{~b}$ SLCC2755 [23], and was conserved in all sequenced ST87 strains. The other CRISPR locus followed by csn2, cas 2 , cas 1 and cas9, which is inserted into the same location (lmo2595) as the reference strain EGD-e (Fig. 1). Complete CRISPR locus was only found in half of the sequenced ST87 strains (35/71), but the gene cas 9 was found in all sequenced strains. The incomplete CRISPR locus in some of the genomes is probably due to gaps in the genome assembly. For 35 ST87 strains with an intact CRISPR locus sequence, a total of 85 spacers and 22 unique spacer arrangements were observed, with spacer numbers between 29 and 40. The spacer content of the CRISPR locus in the ST87 strains was different from strains SLCC5850 (serotype 1/2a), SLCC2540 (serotype 3b), SLCC2755 (serotype 1/2b) and SLCC2482 (serotype 7 ), which had been reported by Kuenne et al [23]. The spacer profiles of each strain were presented in Fig. 2. CRISPR loci evolve via polarized addition of new spacers, or via internal deletion of spacers, or duplication of spacers to create variant profiles. The ST87 CRISPR profiles of spacer-distribution can be classified into two clusters, each of which has a major type represented by seven and nine strains, respectively. One minor type was represented by two strains, and the remaining types were represented by one strain.

\section{ST87-specific gene cluster}

A ST87-specific gene cluster that harbored a type II restriction-modification (RM) system was identified and is present in all ST87 strains sequenced in this study (Fig. 1). The gene cluster was approximately $10 \mathrm{~Kb}$ in size encoding 10 open reading frames (ORFs) and inserted between the gene encoding homoserine dehydrogenase and the gene encoding $50 \mathrm{~S}$ ribosomal protein L31 (Additional file3: Figure S3). The first ORF encodes a protein belonging to an AIPR superfamily which was identified as an abortive infection phage resistance protein often found in RM system operons [26]. The restriction endonuclease recognition site was predicted to be $\mathrm{GCSGC}(\mathrm{S}=\mathrm{C}$ or $\mathrm{G})$ by BLASTN search using REBASE. The DNA methyltransferase, involved in attaching methyl group to C-5 cytosine, exhibited $86 \%$ nucleotide identity and $89 \%$ amino acid identity with a homologue in Enterococcus faecium strain T110 (locus tag: M395_10120). The GC content of the endonuclease gene, the methyltransferase gene and the whole gene cluster were 34.66, 33.16 and $33.78 \%$, respectively, noticeably lower than the genome average of $38 \%$. The gene cluster may have been acquired by horizontal gene transfer, probably from Enterococcus faecium.

\section{Virulence genes and other genetic elements in ST87 $L$. monocytogenes strains}

All well-known virulence factors, including the Listeria pathogenicity island (LIPI)-1, iap, fbpA [27], lpeA [28], lap [29] and $\operatorname{lapB}$ [30] were found in all sequenced ST87 strains. All the sequenced ST87 strains contained multiple internalins, including internalin A, B, C, C2, D, E, $\mathrm{F}, \mathrm{I}, \mathrm{J}$, and $\mathrm{K}$. Also, there were no premature stop codons (PMSC) in inlA in any of the ST87 strains. PMSCs of inlA have often been found in L. monocytogenes isolates from food products but not human cases [20].

All ST87 strains studied harbored the newly reported LIPI-4 pathogenicity island [7], but not LIPI-3, Stress survival islet (SSI)-1 and SSI-2 [8-10]. Instead a LmoOf2365_0481 homolog is present at the location of SSI-1/SSI-2. The pairwise SNP differences in LIPI-4 among strain ICDC-LM188 (ST87), CLIP80459 (ST4), and CFSAN23463 (ST382) were eight or nine SNPs. The upstream flanking sequences of LIPI-4 in ICDC-LM188 and CFSAN23463 were the same, but differed by one SNP from CLIP80459, while the downstream flanking sequences among the three strains were identical. Interestingly, a lineage IV strain J1-208 also harbored LIPI-4, which differed by 223 SNPs from ICDC-LM188. The upstream and downstream sequences differed by 8 and 9 SNPs from ICDC-LM188, respectively (Additional file 4: Figure S4). The putative integration site of LIPI-4 was identified based on a 79-bp sequence with $95 \%$ nucleotide identity between LIPI- $4^{+}$strains and LIPI- $4^{-}$strains (the sequence marked in red in Fig. 4). In Escherichia coli, palindromes are sites of DNA double-strand break formations and the breaks can be repaired by RecBCD pathway through homologous recombination [31]. For LIPI $-4^{+}$L. monocytogenes, a perfect palindrome (the sequence framed in red in Fig. 4) was observed in the intergenic sequence between the LIPI-4 and its next gene, while for LIPI- $4^{-}$strains, an interrupted palindrome (the sequence boxed in blue in Fig. 4) was observed next to the putative integration site (Fig. 4). It has been suggested that the perfect palindrome might be the key to the acquisition of LIPI-4 [31].

\section{Discussion}

In this study, 71 L. monocytogenes ST87 strains were sequenced to investigate the genetic features and relationships of strains of this prevalent ST in China. The 71 strains were divided into 10 clades. The clinical strains spread across multiple branches, suggesting that all ST87 strains can cause human infections. The mutation rate of L. monocytogenes ST87 was estimated to be $4.3 \times 10^{-7}$ substitutions per site per year which was similar to the estimate from a global L. monocytogenes study [32]. The Chinese ST87 strains did not cluster separately from nonChinese ST87. The mixed-distribution of non-Chinese 


\section{SLCC2755}

\section{GCAATACCTCTTTTCGTATGTTAATAGTTTAATTATAGCATTTCTTTAATAAGAAGGCGCCCCATCGCGTAACAAAAAA}

\section{GCAAGATTCCCATTTTCAGGAAACCTTGCTTTTAATTTGT}

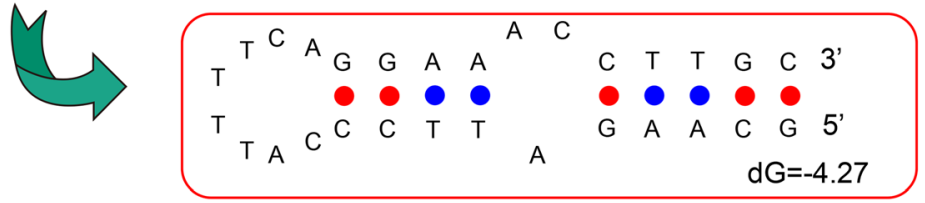

puptative insertion site

ICDC-LM188

GCAATACCTCTTTTCGTATGTTAATAGTTTAATTATAGCATTTCTTTGCAGGGAAGGCGCCCGGTTGGATAACAAAAAA

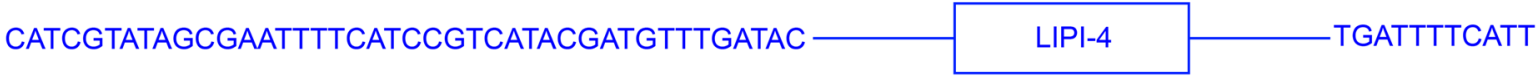

TCCTTTCCCTAAGCTGTTATACGTTCATGATAGCGCATACAAAATTCACCCTCAATTAATCTTATTTCCTAAGCATAGGAA

ATTGTCACTTTACCTTTTTTATACAAAAAACCCCTATAAAGTAATTGCTACTTTATAGGGGTTTTATTTGT

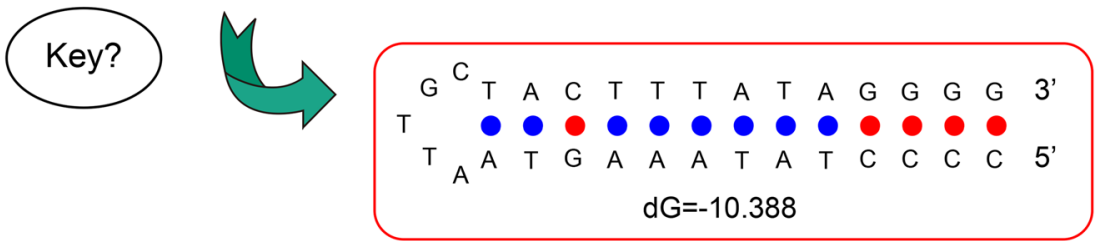

Fig. 4 The flanking sequences of LIPI-4 in strain ICDC-LM188 (LIPI- $\left.4^{+}\right)$and the corresponding sequence in SLCC2755 (LIPI-4 $)$. The identical sequences in both LIPI-4 positive and negative strains were shown in red (upstream) and blank (downstream). The part different was shown in blue. The stem-loop structures of the sequences framed in red were shown below

and Chinese ST87 strains suggests that ST87 has spread to other part of the world since it emerged, presumably, in China. This is not surprising as multiple globally distributed sublineages of $L$. monocytogenes have been found in the recent global L. monocytogenes study [32].

This study also included 10 strains from a one-year longitudinal investigation of $L$. monocytogenes contamination in retailing markets in Zigong, Sichuan province during 2014 to $2015[15,33]$. Among the 10 strains from that study, five strains (LM1249, LM1459, LM1509, LM1605 and LM1637) were isolated from the same booth in February, April to July, 2015, respectively. While another five strains (LM1496, LM1514, LM1515, LM1523 and LM1542) were isolated from different booths which were located in the same market in May, 2015. All of the 10 strains were clustered into one clade (Clade 10) on the phylogenetic tree. Isolations of closely related strains over multiple sampling times in the same market indicated that this clone had persisted in the market environment and was likely to have served as the ultimate source of food contamination in the retail market.

Several previous genomic studies showed that genomic diversity of $L$. monocytogenes was mostly due to mobile genetic elements, such as prophages, transposons, and genetic islands [23]. Our study also identified prophages as the major source of genomic diversity in ST87 $L$. monocytogenes. A total of 11 prophage integration sites (Fig. 1), with 15 prophage profiles (Table 2) were identified. Prophages contributed to a significant proportion of ST87 accessory genome with 10 prophages variably present in different strains. Interestingly LM0097 has no prophages in its genome. It is interesting to note that LM0097 also has the least accessory genes, with $93.7 \%$ of the genomic genes belonging to the core. Prophages P2 and P3 were present in most strains (Fig. 2). The absence of P2 and P3 in some strains is probably due to loss of the prophages.

Noteablely, P3 inserted in the $\operatorname{comK}$ site which is occupied by prophages A118 and $\phi 10403 \mathrm{~S}$ in other strains [34]. Further, excision of the prophage at the $\operatorname{comK}$ site plays an active role as a regulatory switch of $L$. monocytogenes in enhancing niche adaptation, and facilitating escape from phagosomes during mammalian cell infection [34-36]. P3 shared no homology with prophages, A118 and $\phi 10403 S$, previously found in the comK site.

A conserved plasmid was present in 23 of the 71 ST87 L. monocytogenes strains. All of the plasmids from the ST87 strains in this study shared more than $99.9 \%$ sequence similarity to pLM1686 [16]. Plasmid pLM1686 belonged to Listeria plasmids repA group 2, which harbored large plasmids with sizes ranging from $55 \mathrm{~kb}$ to 100 $\mathrm{kb}$ [37]. The plasmid showed very closed relationship with 
Table 2 The profiles of prophage insertion site in each ST87 L. monocytogenes

\begin{tabular}{lllllllllll}
\hline type & IS2 & IS3 & IS4 & IS5 & IS6 & IS7 & IS8 & IS9 & IS10 & IS11 \\
tRNA-Arg & comk & rumA & tsf & mat & $\begin{array}{l}50 S \text { rRNA } \\
\text { methyltransferase }\end{array}$ & tRNA-Lys & tRNA-Ser & tRNA-Arg & tRNA-Thr \\
\hline 1 & & & & & & & & & &
\end{tabular}

ICDC-LM188, ICDC-LM0200, ICDC-LM0216,
ICDC-LM0263, ICDC-LM0322, ICDC-LM0403,
ICDC-LM0417, ICDC-LM0422, ICDC-LM0429,
ICDC-LM0452, ICDC-LM0453, ICDC-LM0476,
ICDC-LM0484, ICDC-LM0725, ICDC-LM0925,
ICDC-LM1074, ICDC-LM1175, ICDC-LM1233,
ICDC-LM1361, ICDC-LM1523, ICDC-LM1620,
ICDC-LM1686

ICDC-LM0078, ICDC-LM0158, ICDC-LM544, ICDC-LM1459

ICDC-LM0077, ICDC-LM0143, ICDC-LM0159, ICDC-LM0250, ICDC-LM1197, ICDC-LM1249, ICDC-LM1496, ICDC-LM1509, ICDC-LM1541, ICDC-LM1515, ICDC-LM1534, ICDC-LM1542, ICDC-LM1551, ICDC-LM1605, ICDC-LM1637, ICDC-LM1784

ICDC-LM0007, ICDC-LM0053, ICDC-LM0111, ICDC-LM0658, ICDC-LM1203, ICDC-LM1204, ICDC-LM1220, ICDC-LM1572, ICDC-LM1674

$\begin{array}{lllllll}5 & + & - & - & - & - & - \\ 6 & - & + & - & - & - & - \\ 7 & + & + & - & + & - & - \\ 8 & - & - & + & - & - & - \\ 9 & - & + & + & - & - & - \\ 10 & + & + & - & - & + & - \\ 11 & + & + & - & - & - & - \\ 12 & - & + & - & - & - & + \\ 13 & - & + & - & - & - & - \\ 14 & - & - & - & - & - & - \\ 15 & - & - & - & - & - & -\end{array}$

\begin{tabular}{|c|c|c|c|c|}
\hline- & - & + & - & $\begin{array}{l}\text { ICDC-LM0007, ICDC-LM0053, ICDC-LM0111, } \\
\text { ICDC-LM0658, ICDC-LM1203, ICDC-LM1204, } \\
\text { ICDC-LM1220, ICDC-LM1572, ICDC-LM1674 }\end{array}$ \\
\hline- & - & + & - & ICDC-LM0099, ICDC-LM1296 \\
\hline- & - & + & - & ICDC-LM0138 \\
\hline- & - & + & - & ICDC-LM0146 \\
\hline - & - & - & - & $\begin{array}{l}\text { ICDC-LM0106, ICDC-LM0108, ICDC-LM0336, } \\
\text { ICDC-LM0915 }\end{array}$ \\
\hline- & - & - & - & ICDC-LM1016, ICDC-LM1117 \\
\hline- & - & - & - & ICDC-LM0441, ICDC-LM0449 \\
\hline+ & - & - & - & ICDC-LM1520 \\
\hline - & - & - & - & $\begin{array}{l}\text { ICDC-LM1681, ICDC-LM1682, ICDC-LM1685, } \\
\text { ICDC-LM1689 }\end{array}$ \\
\hline- & + & - & - & ICDC-LM0402 \\
\hline- & - & - & + & ICDC-LM0208 \\
\hline- & - & - & - & ICDC-LM0097 \\
\hline
\end{tabular}

pSHL004 from strain SHL004, which belonged to CC8 serotype 1/2a from China [38].

Most plasmid-containing strains (18/23) were derived from food products and environments from four retail markets during a 12-month longitudinal investigation in Zigong, Sichuan province [15]. It is possible that maintaining the plasmid may represent a competitive advantage for these strains. The plasmid contained cadmium resistance cadAC genes and also a putative multicopper oxidase gene and copper transporter gene $\operatorname{cop} B$ [16]. A recent study of 3 plasmids, p4KSM, pLMR479a and pLM6179 from 3 different STs, ST5, ST8 and ST121, found that these plasmids contribute to tolerance against elevated temperature, salinity, acidic environments, oxidative stress and disinfectants [39]. These plasmids belonged to the same repA group 2 as the plasmids from our study. All three plasmids contained the cadmium resistance $\operatorname{cadAC}$ genes and one plasmid, pLMR479a, additionally carried a putative multicopper oxidase gene and copper transporter gene $\operatorname{copB}$ similar to pLM1686 [16]. Therefore, carriage of pLM1686 or pLM1686-like plasmid is likely to be advatantagous in surviving the envirotnment.

CRISPR/Cas system is a prokaryotic immune system that confers resistance to foreign DNA, e.g., a virus or plasmid. In the case of Salmonella typhimurium, the profile of spacer arrangements has strong association with strain phylogeny [40]. For ST87 strains, the CRISPR spacer patterns have no correlation with strain phylogeny. Two major types were observed. Strains carried one or the other major type but not both and the distribution of the major types was not clustered. For example, strains ICDC-LM0077 and ICDC-LM1074 were closely related (differing by 52 SNPs) but harbored entirely different sets of spacers. In contrast, strains ICDCLM0097 and ICDC-LM0099 were distantly related to each other (differing by 294 SNPs), but harbored exactly the same set of spacers. There seem to be no 
phylogenetic patterns of the distribution of these two major CRISPR spacer types when compared with the genome tree.

Our study also shed light on the virulence and adaptation of ST87 at genomic level. A ST87 specific gene cluster that is conserved in all ST87 strains was found in this study which carried a novel RM system. RM systems have a diverse range of functions including playing a role in virulence and adaptation to hostile environments [41, 42]. Further studies are warranted to determine whether this novel RM system assists ST87 in survival in the environment and/or virulence in humans. All ST87 strains also carried LIPI-4, a cluster of six genes encoding a cellobiose-family phosphotransferase system (PTS), which has been identified as a virulence factor implicated in maternal-neonatal (MN) and central nervous system (CNS) infections previously [7, 22]. ST87 is prevalent in causing human infections in China and the presence of LIPI-4 may have contributed to its prevalence, which underscores the importance of epidemiological surveillance of ST87 from food sources and human infections in China.

\section{Conclusion}

ST87 L. monocytogenes strains were frequently isolated from food products, environments and human clinical infections in China. The ST87 core genome carried 2677 genes. All ST87 strains carried prophage P1 and variably 10 other prophages as a major component of the ST87 accessory genome. ST87 contained a novel type II RM system although its significance is unknown, and a virulent gene cluster LIPI-4. A novel plasmid was present in a small proportion of the ST87 strains and nearly all were from food products or environments from four retail markets from a single region. The plasmid may offer the host an advantage to compete and persist in certain environments. ST87 arose 200 years ago and expanded rapidly in its early evolution in China. This study has shed light on the genomic diversity, evolution, adaptation and pathogenicity of ST87, the predominant ST that causes human infections in China.

\section{Methods}

\section{Strains used for genome sequencing and DNA isolation}

A total of 71 ST87 L. monocytogenes strains were used. The isolates were obtained from 2001 to 2015 from 10 regions of China including nine isolates from human infections, 47 from food sources and 11 from the environment (Additional file 5: Table S1). The strains were stored in Brain Heart Infusion (BHI) broth containing $15 \%$ glycerol at $-80^{\circ} \mathrm{C}$, and cultured on $\mathrm{BHI}$ agar at $37^{\circ} \mathrm{C}$. Genomic DNA from pure cultures was extracted using the Wizard ${ }^{\circ}$ Genomic DNA Purification Kit (cat. \# A1120, Promega, USA), according to the manufacturer's instructions for Gram-positive bacteria.

\section{Genome sequencing, assembly and annotation}

The whole genome of L. monocytogenes strain ICDCLM188 was determined by using the Illumina HiSeq 2000 platform. The reads were assembled de novo into a high-quality draft genome using Velvet (version 1.2.10) with default parameters and with an average 85 -fold sequencing coverage. All of the gaps were closed by primer walking and sequencing of PCR products using Applied Biosystems 3730 DNA Analyzer. Genome annotation was performed using the NCBI Prokaryotic Genomes Automatic Annotation Pipeline (PGAAP). Whole-genome shotgun sequencing of the other 70 ST87 L. monocytogenes strains was performed on the Illumina HiSeq X PE150 platform. The read quality was assessed with FastQC, and the reads were filtered to remove low-quality sequences (quality score $\leq 38$ ), ambiguous reads (reads with 10 or more unknown base N) and adapter sequences. High-quality paired-end reads were assembled into scaffolds using SOAPdenovo v1.05. The coding genes were retrieved using the GeneMarkS (version 4.17, http://topaz.gatech.edu/)program [43] or Rapid Annotations based on Subsystem Technology (RAST) server (version 2, http://rast.nmpdr.org/rast.cgi) [44]. The database of Clusters of Orthologous Groups of proteins (COGs) was used to predict gene functions and the parameters for BLASTP searches were E-value of less than $10^{-5}$ and larger than $40 \%$ of the query coverage.

\section{Core-pan genome analysis}

Identification of homologous proteins was performed by the CD-HIT rapid clustering of similar proteins software [45], based on a similarity threshold of $50 \%$ amino acid identity and $70 \%$ coverage.

\section{Detection of the core genome SNPs}

For the detection of SNPs, Illumina reads from the 70 ST87 strains sequenced were mapped against the closed genome of $L$. monocytogenes strain ICDC-LM188 using Burrows-Wheeler Aligner (BWA-MEM) [46]. SNP calls were performed by SAMtool (version 0.1.19) followed by BCFtools 1.9, and a vcf file for each isolate was generated. High-quality SNPs were identified using the following criteria: i). a minimum coverage of 20 reads; and ii). minimum variant frequency of $70 \%$ [47]. We searched against the COG database to catagorise the core genes of ST87.

\section{Phylogenetic analysis based on the whole genomic SNPs}

The SNPs located in the core genome were used to perform phylogenetic analysis on all sequenced $L$. monocytogenes ST87 strains. The SNPs for each genome was concatenated to a multi-alignment FASTA file, and then imported to MEGA6 to generate a maximum likelihood tree using default parameters. For further evolutionary analysis of ST87 strains, phylogenetic inference was 
performed with Bayesian Evolutionary Analysis by Sampling Trees (BEAST, v2.4.7). BEAST analysis was performed with three replicates. The best-fit evolutionary analysis was performed under GTR model of substitution, along with a relaxed clock log normal clock model and Birth Death Model tree prior. We performed the run for 50,000,000 generations, sampling every 1000 generations and analyzed the output by using the Tracer module with all ESS values $>200$. The burnin percentage was set to 10 for Markov chain Monte Carlo (MCMC) output analysis by TreeAnnotator (v2.4.7).

\section{Analysis of prophage, plasmids, CRISPR, LIPI-4 and virulence genes}

Prophage regions were identified using PHASTER with default parameters online (http://phaster.ca) and were considered to be incomplete, questionable or complete when its completeness score was less than 60 , between 60 and 90 or more than 90 , respectively [48]. The plasmids in ST87 strains were identified by BLASTN searches using the sequence of pLM1686 as query against each assembled sequence. For the comparative analysis of pLM1686 with other L. monocytogenes plasmids, RepA protein sequences were used to infer their phylogenetic relationships by the Maximum Likelihood method. The CRISPR system was identified by CRISPRFinder from https://crispr.i2bc.parissaclay.fr/Server/ [49]. The divergence of LIPI-4 and flanking sequences in L. monocytogenes ICDC-LM188, CFSAN023463, CLIP80459 and J1-208 was determined using MAUVE and BLASTN, respectively. The presence/ absence of LIPI-3, stress survival islets SSI-1 and SSI-2 and other virulence genes were determined using BLASTN with a threshold of $\geq 60 \%$ query coverage with $\geq 80 \%$ nucleic acid sequence identity.

\section{Supplementary information}

Supplementary information accompanies this paper at https://doi.org/10. 1186/s12864-019-6399-1.

\footnotetext{
Additional file 1: Figure S1. COG analysis of core genes (A) and accessory genes (B). COG categories are as follows: C: Energy production and conversion, D: Cell cycle control, cell division, chromosome partitioning, E: Amino acid transport and metabolism, F: Nucleotide transport and metabolism, G: Carbohydrate transport and metabolism; H: Coenzyme transport and metabolism, l: Lipid transport and metabolism, $\mathrm{J}$ : Translation, ribosomal structure and biogenesis, L: Transcription, K: Replication, recombination and repair, M: Cell wall/membrane/envelope biogenesis, N: Cell motility, O: Posttranslational modification, protein turnover, chaperones, P: Inorganic ion transport and metabolism, Q: Secondary metabolites biosynthesis, transport and catabolism, R: General function prediction only; S: Function unknown; T: Signal transduction mechanisms; U: Intracellular trafficking, secretion, and vesicular transport; V: Defense mechanisms, W: Extracellular structures, X: Mobilome, prophages, transposons

Additional file 2: Figure S2. Maximum likelihood phylogenetic tree of Chinese and non-Chinese ST87 strains. The phylogenetic analysis was performed based on the SNPs located in the core genes of ST87 L. monocytogenes. The non-Chinese strains were colored blue.
}

Additional file 3: Figure S3. ST87-specific gene cluster. The putative function of each ORF is shown. The flanking genes of the cluster are in green. The two major components of the R-M system coding genes are in blue, while the other genes of this cluster are in red.

Additional file 4: Figure S4. The alignments of flanking intergenic sequences of LIPI-4. A for upstream region (located in ICDC-LM188 from $2,440,881$ to $2,441,001$ ) and B for downstream region (located in ICDCLM188 from 2,447,012 to 2,447,174). L. monocytogenes strains ICDC-LM188 (ST87, Lineage I), CFSAN023463 (ST382, Lineage I), CLIP80459 (ST4, Lineage I) and J1-208 (ST unknown, Lineage IV).

Additional file 5: Table S1. List of ST87 L. monocytogenes strains used in this study.

Additional file 6: Table S2. Summary statistics of whole genome assemblies and gene prediction.

\section{Abbreviations}

CC: Clonal complex; LIPI: Listeria pathogenicity island; MLST: Multi-locus sequence typing; RM system: Restriction-modification system; SNP: Single nucleotide polymorphism; SSI: Stress survival islet; ST: Sequence type; WGS: Whole genome sequencing

\section{Acknowledgments}

The authors thank Ying Jiao, Xuebing Xu and Genyan Liu for providing clinical L. monocytogenes strains and the Centers for disease control and prevention of Fujian province, Hanzhou, Shenzhen and Wuhan city for providing the L. monocytoegens ST87 strains from food products and environments.

\section{Authors' contributions}

YW, RL, JX and CY conceived and designed the research; LL, QL and HW isolated and characterised the majority of the ST87 strains used; YW, YQW and HS performed the experiments and contributed for raw data analysis. YW, LL and RL contributed in the bioinformatics analysis; YW, RL and CY wrote and revised the paper. All authors read and approved the final manuscript.

\section{Funding}

This work was supported by grants from State Key Laboratory of Infectious Disease Prevention and Control, China CDC (2018ZZKTB07 and 2018SKLID801) and from National Natural Science Foundation of China (31800004). The funding bodies played no role in the design of the study and collection, analysis, and interpretation of data and in writing the manuscript.

\section{Availability of data and materials}

The datasets generated and/or analysed during the current study are included in this published article. The genome sequences are deposited in NCBI database. The complete genome ICDC-LM188 has been deposited in DDBJ/ENA/GenBank under the accession number CP015593. The draft genome sequences of the other strains sequenced in this study has been deposited at DDBJ/ENA/Genbank under the BioProject accession number PRJNA447903. The accession numbers of LM1686 and pLM1686 are SAMN10373576 and MK134858, respectively. Publicly available raw sequence data of seven non-Chinese ST87 strains were included in the comparative analysis between the non-Chinese and Chinese ST87 L. monocytogenes. Six genomes were from NCBI's SRA database with accession numbers for six sequences are SRR1112090, SRR1112227, SRR946037, SRR972401, SRR972405, SRR974856 and one was obtained from ENA with the accession number ERS1044951.

Ethics approval and consent to participate

Not applicable.

\section{Consent for publication}

Not applicable.

\section{Competing interests}

The authors declare that they have no competing interests. 


\section{Author details}

${ }^{1}$ State Key Laboratory of Infectious Disease Prevention and Control, National Institute for Communicable Disease Control and Prevention, Collaborative Innovation Center for Diagnosis and Treatment of Infectious Diseases, Chinese Center for Disease Control and Prevention, Beijing 102206, China. ${ }^{2}$ School of Biotechnology and Biomolecular Sciences, University of New South Wales, Sydney, NSW 2052, Australia. ${ }^{3}$ Zigong Center for Disease Control and Prevention, Zigong 643000, Sichuan Province, China.

\section{Received: 3 March 2019 Accepted: 15 December 2019} Published online: 23 December 2019

\section{References}

1. Toledo-Arana A, Dussurget O, Nikitas G, Sesto N, Guet-Revillet H, Balestrino D, Loh E, Gripenland J, Tiensuu T, Vaitkevicius K, et al. The Listeria transcriptional landscape from saprophytism to virulence. Nature. 2009; 459(7249):950-6.

2. Liu D. Handbook of Listeria monocytogenes. Boca Raton: CRC press; 2008.

3. Aureli P, Fiorucci GC, Caroli D, Marchiaro G, Novara O, Leone L, Salmaso S. An outbreak of febrile gastroenteritis associated with corn contaminated by Listeria monocytogenes. N Engl J Med. 2000;342(17):1236-41.

4. Angelo KM, Conrad AR, Saupe A, Dragoo H, West N, Sorenson A, Barnes A, Doyle M, Beal J, Jackson KA, et al. Multistate outbreak of Listeria monocytogenes infections linked to whole apples used in commercially produced, prepackaged caramel apples: United States, 2014-2015. Epidemiol Infect. 2017:1-9.

5. Thomas MK, Vriezen R, Farber JM, Currie A, Schlech W, Fazil A. Economic cost of a Listeria monocytogenes outbreak in Canada, 2008. Foodborne Pathog Dis. 2015;12(12):966-71.

6. Orsi RH, HCd B, Wiedmann M. Listeria monocytogenes lineages: genomics, evolution, ecology, and phenotypic characteristics. Int J Med Microbiol. 2011;301(2):79-96.

7. Maury MM, Tsai YH, Charlier C, Touchon M, Chenal-Francisque V, Leclercq A, Criscuolo A, Gaultier C, Roussel S, Brisabois A, et al. Uncovering Listeria monocytogenes hypervirulence by harnessing its biodiversity. Nat Genet. 2016;48(3):308-13.

8. Cotter PD, Draper LA, Lawton EM, Daly KM, Groeger DS, Casey PG, Ross RP, Hill C. Listeriolysin S, a novel peptide haemolysin associated with a subset of lineage I Listeria monocytogenes. PLoS Pathog. 2008;4(9):e1000144.

9. Ryan S, Begley M, Hill C, Gahan CG. A five-gene stress survival islet (SSI-1) that contributes to the growth of Listeria monocytogenes in suboptimal conditions. J Appl Microbiol. 2010;109(3):984-95.

10. Harter E, Wagner EM, Zaiser A, Halecker S, Wagner M, Rychli K, Drake HL. Stress survival islet 2, predominantly present in Listeria monocytogenes strains of sequence type 121, is involved in the alkaline and oxidative stress responses. Appl Environ Microbiol. 2017;83(16):e00827-17.

11. Wu S, Wu Q, Zhang J, Chen M, Yan Z. Prevalence, antibiotic resistance and genetic diversity of Listeria monocytogenes isolated from retail ready-to-eat foods in China. Food Control. 2015;47:340-7.

12. Wang G, Qian W, Zhang X, Wang H, Ye K, Bai Y, Zhou G. Prevalence, genetic diversity and antimicrobial resistance of Listeria monocytogenes isolated from ready-to-eat meat products in Nanjing, China. Food Control. 2015;50:202-8.

13. Wang $Y$, Zhao A, Zhu R, Lan R, Jin D, Cui Z, Wang Y, Li Z, Wang Y, Xu J, et al. Genetic diversity and molecular typing of Listeria monocytogenes in China. BMC Microbiol. 2012;12:119.

14. Wang $Y$, Jiao $Y$, Lan $R$, Xu X, Liu G, Wang X, Zhang L, Pang H, Jin D, Dai H, et al. Characterization of Listeria monocytogenes isolated from human Listeriosis cases in China. Emerg Microbes Infect. 2015:4:e50.

15. Luo L, Zhang Z, Wang H, Wang P, Lan R, Deng J, Miao Y, Wang Y, Wang Y, $\mathrm{Xu}$ J. A 12-month longitudinal study of Listeria monocytogenes contamination and persistence in pork retail markets in China. Food Control. 2017:66-73.

16. Luo L, Chen X, Payne M, Cao X, Wang Y, Zhang J, Deng J, Wang H, Zhang $Z$, Li Q, et al. Case report: whole genome sequencing based investigation of maternal-neonatal listeriosis in Sichuan, China. BMC Infect Dis. 2019;19(1):893.

17. Huang YT, Ko WC, Chan YJ, Lu JJ, Tsai HY, Liao CH, Sheng WH, Teng LJ, Hsueh PR. Disease burden of invasive listeriosis and molecular characterization of clinical isolates in Taiwan, 2000-2013. PLoS One. 2015; 10(11):e0141241.
18. Li W, Bai L, Fu P, Han H, Liu J, Guo Y. The epidemiology of Listeria monocytogenes in China. Foodborne Pathog Dis. 2018;15(8):459-66.

19. Perez-Trallero E, Zigorraga C, Artieda J, Alkorta M, Marimon JM. Two outbreaks of Listeria monocytogenes infection, Northern Spain. Emerg Infect Dis. 2014;20(12):2155-7.

20. Schmitz-Esser S, Müller A, Stessl B, Wagner M. Genomes of sequence type 121 Listeria monocytogenes strains harbor highly conserved plasmids and prophages. Front Microbiol. 2015;6:380.

21. Fox EM, Allnutt T, Bradbury MI, Fanning S, Chandry PS. Comparative genomics of the Listeria monocytogenes ST204 subgroup. Front Microbiol. 2016;7:2057.

22. Chen Y, Luo Y, Pettengill J, Timme R, Melka D, Doyle M, Jackson A, Parish M, Hammack TS, Allard MW, et al. Singleton sequence type 382, an emerging clonal group of Listeria monocytogenes associated with three multistate outbreaks linked to contaminated stone fruit, caramel apples, and leafy green salad. J Clin Microbiol. 2017;55(3):931-41.

23. Kuenne C, Billion A, Mraheil MA, Strittmatter A, Daniel R, Goesmann A, Barbuddhe S, Hain T, Chakraborty T. Reassessment of the Listeria monocytogenes pan-genome reveals dynamic integration hotspots and mobile genetic elements as major components of the accessory genome. BMC Genomics. 2013;14:47.

24. Zhou Y, Liang Y, Lynch KH, Dennis JJ, Wishart DS. PHAST: a fast phage search tool. Nucleic Acids Res. 2011;39(Web Server issue):W347-52.

25. Furuta $Y$, Abe K, Kobayashi I. Genome comparison and context analysis reveals putative mobile forms of restriction-modification systems and related rearrangements. Nucleic Acids Res. 2010;38(7):2428-43.

26. lyer LM, Abhiman S, Aravind L. MutL homologs in restriction-modification systems and the origin of eukaryotic MORC ATPases. Biol Direct. 2008;3:8.

27. Dramsi S, Bourdichon F, Cabanes D, Lecuit M, Fsihi H, Cossart P. FbpA, a novel multifunctional Listeria monocytogenes virulence factor. Mol Microbiol. 2004:53(2):639-49.

28. Reglier-Poupet H, Pellegrini E, Charbit A, Berche P. Identification of LpeA, a PsaA-like membrane protein that promotes cell entry by Listeria monocytogenes. Infect Immun. 2003;71(1):474-82.

29. Burkholder KM, Bhunia AK. Listeria monocytogenes uses Listeria adhesion protein (LAP) to promote bacterial transepithelial translocation and induces expression of LAP receptor Hsp60. Infect Immun. 2010;78(12):5062-73.

30. Reis O, Sousa S, Camejo A, Villiers V, Gouin E, Cossart P, Cabanes D. LapB, a novel Listeria monocytogenes LPXTG surface adhesin, required for entry into eukaryotic cells and virulence. J Infect Dis. 2010;202(4):551-62.

31. Eykelenboom JK, Blackwood JK, Okely E, Leach DR. SbcCD causes a doublestrand break at a DNA palindrome in the Escherichia coli chromosome. Mol Cell. 2008;29(5):644-51.

32. Moura A, Criscuolo A, Pouseele H, Maury MM, Leclercq A, Tarr C, Bjorkman JT, Dallman T, Reimer A, Enouf V, et al. Whole genome-based population biology and epidemiological surveillance of Listeria monocytogenes. Nat Microbiol. 2016;2:16185.

33. Wang H, Luo L, Zhang Z, Deng J, Wang Y, Miao Y, Zhang L, Chen X, Liu X, Sun $\mathrm{S}$, et al. Prevalence and molecular characteristics of Listeria monocytogenes in cooked products and its comparison with isolates from listeriosis cases. Front Med. 2018;12(1):104-12.

34. Rabinovich L, Sigal N, Borovok I, Nir-Paz R, Herskovits AA. Prophage excision activates Listeria competence genes that promote phagosomal escape and virulence. Cell. 2012;150(4):792-802.

35. Verghese B, Lok M, Wen J, Alessandria V, Chen Y, Kathariou S, Knabel S. comK prophage junction fragments as markers for Listeria monocytogenes genotypes unique to individual meat and poultry processing plants and a model for rapid niche-specific adaptation, biofilm formation, and persistence. Appl Environ Microbiol. 2011;77(10):3279-92.

36. Feiner R, Argov T, Rabinovich L, Sigal N, Borovok I, Herskovits AA. A new perspective on lysogeny: prophages as active regulatory switches of bacteria. Nat Rev Microbiol. 2015;13(10):641-50.

37. Ahmed N, Kuenne C, Voget S, Pischimarov J, Oehm S, Goesmann A, Daniel R, Hain T, Chakraborty T. Comparative analysis of plasmids in the genus Listeria. PLoS One. 2010;5(9):e12511.

38. Zhang J, Cao G, Xu X, Allard M, Li P, Brown E, Yang X, Pan H, Meng J. Evolution and diversity of Listeria monocytogenes from clinical and food samples in Shanghai, China. Front Microbiol. 2016;7:1138.

39. Naditz AL, Dzieciol M, Wagner M, Schmitz-Esser S. Plasmids contribute to food processing environment-associated stress survival in three Listeria monocytogenes ST121, ST8, and ST5 strains. Int J Food Microbiol. 2019;299:39-46. 
40. Fu S, Hiley L, Octavia S, Tanaka MM, Sintchenko V, Lan R. Comparative genomics of Australian and international isolates of Salmonella Typhimurium: correlation of core genome evolution with CRISPR and prophage profiles. Sci Rep. 2017;7(1):9733.

41. Kim JW, Dutta V, Elhanafi D, Lee S, Osborne JA, Kathariou S. A novel restriction-modification system is responsible for temperature-dependent phage resistance in Listeria monocytogenes ECll. Appl Environ Microbiol. 2012;78(6):1995-2004.

42. Vasu K, Nagaraja V. Diverse functions of restriction-modification systems in addition to cellular defense. Microbiol Mol Biol Rev. 2013;77(1):53-72.

43. Besemer J, Lomsadze A, Borodovsky M. GeneMarkS: a self-training method for prediction of gene starts in microbial genomes. Implications for finding sequence motifs in regulatory regions. Nucleic Acids Res. 2001;29(12):2607-18.

44. Aziz RK, Bartels D, Best AA, DeJongh M, Disz T, Edwards RA, Formsma K, Gerdes S, Glass EM, Kubal M, et al. The RAST server: rapid annotations using subsystems technology. BMC Genomics. 2008;9:75.

45. Li W, Godzik A. Cd-hit: a fast program for clustering and comparing large sets of protein or nucleotide sequences. Bioinformatics. 2006;22(13):1658-9.

46. Li H, Durbin R. Fast and accurate short read alignment with burrowswheeler transform. Bioinformatics. 2009;25(14):1754-60.

47. Pang S, Octavia S, Feng L, Liu B, Reeves PR, Lan R, Wang L. Genomic diversity and adaptation of Salmonella enterica serovar Typhimurium from analysis of six genomes of different phage types. BMC Genomics. 2013;14:718.

48. Arndt D, Grant JR, Marcu A, Sajed T, Pon A, Liang Y, Wishart DS. PHASTER: a better, faster version of the PHAST phage search tool. Nucleic Acids Res. 2016:44(W1):W16-21.

49. Grissa I, Vergnaud G, Pourcel C. CRISPRFinder: a web tool to identify clustered regularly interspaced short palindromic repeats. Nucleic Acids Res. 2007;35(Web Server issue):W52-7.

\section{Publisher's Note}

Springer Nature remains neutral with regard to jurisdictional claims in published maps and institutional affiliations.

Ready to submit your research? Choose BMC and benefit from:

- fast, convenient online submission

- thorough peer review by experienced researchers in your field

- rapid publication on acceptance

- support for research data, including large and complex data types

- gold Open Access which fosters wider collaboration and increased citations

- maximum visibility for your research: over $100 \mathrm{M}$ website views per year

At $\mathrm{BMC}$, research is always in progress.

Learn more biomedcentral.com/submissions 